\title{
REPORT
}

\author{
of the
}

CEBAF

\section{PAC4 SUBCOMMITTEE}

\author{
on \\ STAR
}

\section{DISCLAIMER}

This report was prepared as an account of work sponsored by an agency of the United States Government. Neither the United States Government nor any agency thereof, nor any of their employees, makes any warranty, express or implied, or assumes any legal liability or responsibility for the accuracy, completeness, or usefulness of any information, apparatus, product, or process disclosed, or represents that iss use would not infringe privately owned rights. Reference herein to any specific commercial product, process, or service by trade name, trademark, manufacturer, or othciwise does not necessarily constitute or imply its endorsement, recommendation, or favoring by the United States Government or any agency thereof. The views and opinions of authors expressed herein do not necessarily state or reflect those of the United States Government or any agency thereof.

REVIEW AT CEBAF

FINAL REPORT
תIY $17-18,1990$

Aug 8, 1990 MASTER DISTRIBUTION OF THIS DOCUMIENT IS UNLIMI: 
Professor John Dirk Walecka

Scientific Director, CEBAF

12070 Jefferson Avenue

Newport News, Virginia 23606

Dear Dirk,

Enclosed is the copy of the PAC subcomittee on STAR. I have polled

the PAC and a majority of members have responded, all in favor of accepting

the report for transmission to you.

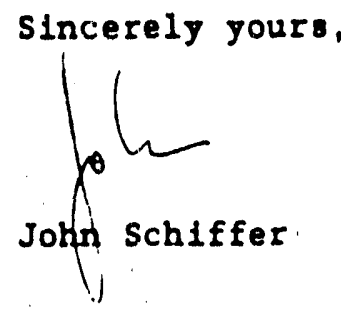

JS : jCB

Enclosure 


\section{Carnegie Mellon}

August 8, 1990

Dr. John P. Schiffer

Physics Division, Bldg. 203

Argonne National Lab.

9700 S. Cass Ave.

Argonne, Illinois, 60439

Dear John, STAR.

Enclosed is the Report of the CEBAF PAC4 Subcommittee for the review of

The possible role and priority of an extensive out-of-plane detection capability at CEBAF has been discussed in the CEBAF community and at a number of PAC meetings. The STAR collaboration, led by Drs. Costas Papanicolas and Ronald Laszewski of the University of Illinois, has developed a broad program of out-of-plane physics based on a very innovative and original magnetic spectrometer design.

We have reviewed this project as requested by PAC4. The Subcommittee was very impressed with the creative and thorough way in which the reference design was worked out and the challenging physics experiments identified for the STAR program. We have treated the docuinents presented as a Letter of Intent rather than as a full proposal. The cost estimates for the project, as presented, were not reviewed in detail.

The major concern of the Subcommittee during the review was whether the impact of the physics program presented would be cornmensurate with the high cos't and the major commitment of manpower required in order to construct this device. Out-or-plane and spin dependent structure function measurements using the STAR would in some sense be second generation experiments. Since both medium-energy measurements and a firstgeneration program at CEBAF will produce results which should provide indications of where the focus for future developments should be placed, we conclucie that it is premature to make a major commitment on the scale of STAR at this time. Our concerns, conclusions, and recommendations are contained in the Eriecutive Summary and in Section VI of the Report.

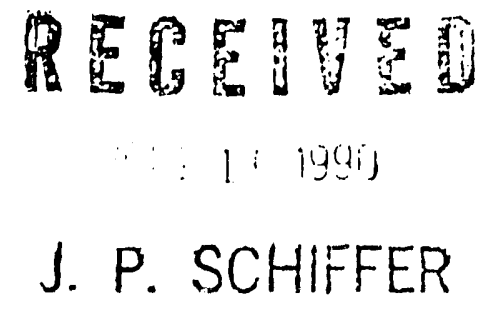


I would like to thank all the members of the STAR collaboration, the CEBAF staff, and the members of this Subcominittee and its consultants for making this an interesting and productive meeting. My special thanks go to Kathy Strozak for her help in organizing the review sessions.

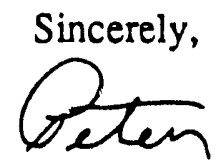

Peter D. Barnes, Chairman

PAC4 Subcommittee on STAR

enclosure

cc:

J. Domingo

C. Papanicolas

J. Dirk Walecka 


\title{
REPORT
}

\author{
of the \\ CEBAF \\ PAC4 SUBCOMMITTEE
}

on

STAR

REVIEW AT CEBAF

FINAL REPORT
ЛルY $17-18,1990$

Aug 8, 1990 


\section{CONTENTS}

PAGE

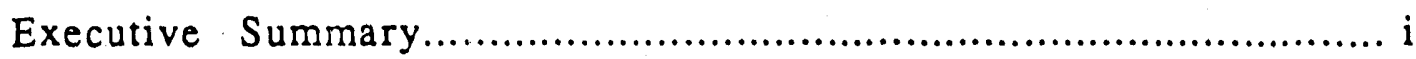

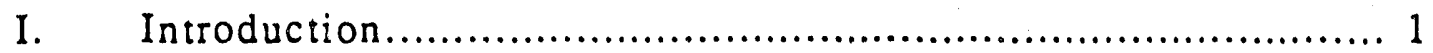

II. The Symmetric Toroidal Array Spectrometer Facility......................... 2

III. Physics Topics......................................................... 4

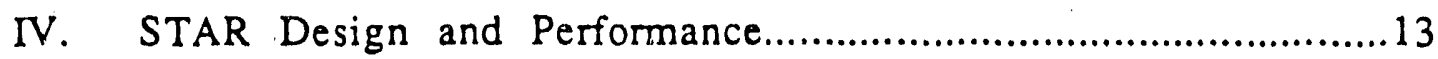

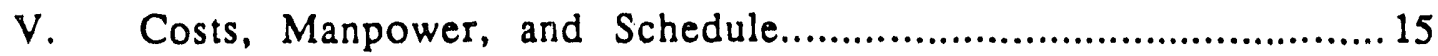

VI. Summary and Recommendation..............................................15

Appendix A: Membership of the PAC4 Subcomn.ittee for the

Review of STAR ............................................................ 19

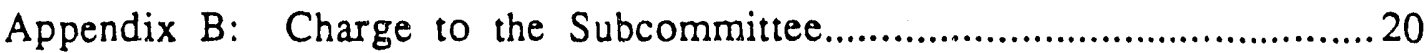

Appendix C: Agenda of the Meetings at CEBAF......................................... 21 


\section{EXECUTIVE SUMMARY}

The CEBAF PAC4 Subcommittee on STAR, the Symmetric Toroidal ARray, performed a detailed review of this spectrometer facility on July $17-18,1990$. This device consists of an eight-sector toroidally symmetric multi-gap spectrometer designed for use at CEBAF. The instrument is intended to operate both in conjunction with an electron spectrometer in the pursuit of programs of out-of-plane electro-coincidence measurements and as a recoil polarimeter. In the first case the multi-gap spectrometer allows the extraction of all five response functions which are available from (e,e'p) reactions with polarized electron beams. As a stand-alone detector, it could also be utilized in parity nonconservation measurements.

The spectrometer is of toroidal design with eight azimuthally-symmetric sectors. Each sector consists of an inward-bending, singly-focusing dipole with a gap width of $40 \mathrm{~cm}$. By shifting the object (target) position, the opening angle, $\theta_{\mathrm{pq}}$, of the particle trajectery relative to the central axis can be varied from 5 to 50 degrees as the acceptance solid angle (eight gaps) shifts from 7.4 to $153 \mathrm{msr}$. The nominal maximum central momentum varies monotonically from 3.1 $\mathrm{GeV} / \mathrm{c}$ at an opening angle of 5 degrees to $1.5 \mathrm{GeV} / \mathrm{c}$ at 50 degrees. The momentum acceptance is $15 \%$ of the central value; the momenturu resolution is $0.1 \%$.

The second arm of the coincidence measurements is expected to be either the HMS or the SOS as described in the CEBAF Conceptional Design Report. The luminosity limit for the system has been estimated to be about $10^{38} \mathrm{~cm}^{-2} \mathrm{sec}^{-1}$. The design goal is to measure spacial and spinflip asymmetries with an absolute error of 0.01 . The device would sit in Hall $\mathrm{C}$ at CEBAF and has a construction cost in excess of $\$ 20 \mathrm{M}$ (including $30 \%$ for contingency) The Subcommittee did not review the details of this cost estimate.

The major thrusts of the STAR program are the investigation of spin dependent and out-of-plane structure functions and the investigation of parity violation phenomena.

At CEBAF, the out-of-plane coincidence program of STAR complements the programs planned for Hall A using polarimetry or the proposed MPS facility, and for Hall B using CLAS. STAR, with its large acceptance and high luminosity capability, is unique in its ability to study small effects at high $\mathrm{Q}^{2}$ and at large recoil momentum. However, the new generation of experiments now planned or in progress at Bates, at Mainz, and developing at CEBAF have the potential for generating major advances in our 
understanding of spin dependent and out-of-plane structure functions. Thus it is difficult to assess, at this time, the incremental impact of the additional information provided by STAR: It is the Subcommittee's judgement that major additions to the CEBAF out-ofplane capability should await developments in the programs at the lower energy facilities.

The investigation of parity nonconserving processes is potentially a very interesting new component of the CEBAF physics program. Although several experiments have been discussed, a specific physics goal for the development of this program has not been established since no clear path to a precision measurement has yet evolved. The relative merits of a program using STAR, the Hall A spectrometers, or a new magnetic device ( for example a solenoid) have been discussed but not worked out. It is the judgeme it of this Subcommittee that the case for basing this we $k$ interaction physics program on the STAR facility has not been made.

\section{Subcommittee Recommendation}

It is the unanimous conclusion of this Subcommittee that, as presented, the incremental physics impact of the STAR program over experiments in progress or planned, is not sufficient to justify an expenditure which we judge to be in excess of $\$ 20 \mathrm{M}$. We do not recommend construction of this device.

The Subcommittee gave some thought as to the merits of focusing on a more limited physics program based on a less expensive but more limited spectrometer. There was no consensus among the Subcommittee members as to what physics could serve as the basis for such a more limited design and w; take no position on this issue. 


\section{INTRODUCTION}

At the March 19-23, 1990 meeting of CEBAF PAC4, a Subcommittee was appointed to respond to a request from the CEBAF management to review the physics case for the construction of a Symmetric Toroidal ARray Spectrometer, STAR, for operation at CEBAF. The Subcommittce held a brief organizational meeting on March 23, and reviewed this project at CEBAF on July 17 and 18, 1990. The membership of the Subcommittee, the names of its three consultants, and the charge fro.n CEBAF management to PAC4 as delegated to the Subcommittee are provided in Appendix $A$ and $B$ of this report. Appendix $C$ contains the agenda of the July 17 18 s zview at CEBAF.

Written materials describing the physics program, the design, and the operation of the spectrometer were presented to the Subcommittee during June and early July, 1990. The Subcommittee chose to treat these materials as a rather detailed Letter of Intent rather than a fully developed experimental proposal. At its review meetings of July 17 and 18, the Subconmittee met in both open arid closed sessions. Presentations by the STAR collaboration, under the leadership of Dr. Costas Papanicolas of the University of Illinois, defined the physics goals of the project. The conceptual design of STAR was presented by Dr. Ronald Laszewski, also of the Univ. of nlinois. Additional critiques of the project and its relationship to the CEBAF program as defined by the CDR, were presented by Drs. R. Lourie, B. Mecking, and P. Souder as requested by the Subcommittee.

The possible role and priority of an out-of-plane detection capability at CEBAF has been discussed at a number of PAC meetings. At its last two meetings the PAC has heard reports from the STAR collaboration on the physics motivation and on the status of their spectrometer design. Over the past year, the STAR collaboration, led by the University of Ilinois group, has developed a broad program of physics based on a very innovative and original magrietic spectrometer design. This Subcommittee was very impressed with the creative and thorough way in which the reference design was worked out and the challenging physics experiments identified for the STAR prom. The major concern of the committee during the presentations was whether the impact of the physics program presented would be commensurate with the high cost and major commitment of manpower required to construct this device.

In this report, an overview of the spectrometer is presented in Section II. The physics program is reviewed in Section III, followed by a discussion of the reference spectrometer design in Section IV. Section V contains a discussion of resource issues. The conclusions and 
recommendations of the Subcommittee are presented in an EXECUTTVE SUMMARY in the first section of this report and in more detail in Section VI.

\section{THE SYMMETRIC TOROIDAL ARRAY SPECTROMETER FACILITY}

This instrument is a multi-purpose spectrometer intended for operation at CEBAF both in conjunction with an electron spectrometer in the pursuit of programs of out-of-plane electrocoincidence measurements and as a recoil polarimeter. In the first case, the multi-gap magnet allows the extraction of all five structure functions which are available from out-of-plane studies of (e,e'p) with polarized electron beams. As a stand-alone detector, it could be utilized in parity nonconservation measurements, for example in the measurement of elastic scaitering of polarized electrons from protons.

The spectrometer is of toroidal design with eight azimuthally-symmetric sectors. Each sector consists of an inward-bending, singly-focusing dipole with a gap width of $40 \mathrm{~cm}$. By shifting the object (target) position, the opening angle, $\theta_{\mathrm{pq}}$, of the particle trajectery relative to the central axis can be varied from 5 to 50 degrees as the acceptance solid angle shifts from 7.4 to $153 \mathrm{msr}$ (eight gaps). The nominal maximum central momentum varies monotonically from 3.1 $\mathrm{GeV} / \mathrm{c}$ at an opening angle of 5 degrees to $1.5 \mathrm{GeV} / \mathrm{c}$ at 50 degrees. The momentum acceptance is $15 \%$ of the central value; the momentum resolution is $0.1 \%$.

The second arm of the coincidence measuremen's is expected to be either the HMS or the SOS as described in the CEBAF Conceptional Design Report. The luminosity limit for the system has been estimated to be about $10^{38} \mathrm{~cm}^{-2} \mathrm{sec}^{-1}$. The design goal is to measure spacial and spinflip asymmetries with an absolute error of 0.01 .

A summary of the gross physical characteristics of the STAR reference design is given in Table I while a summary of the operating characteristics of STAR is given in Table II. 
TABLE I.

STAR Design Parameters

$\begin{array}{lc}\text { B - max } & 20 \mathrm{kG} \\ \text { Gap width } & 40 \mathrm{~cm} \\ \text { \# of gaps } & 8 \\ \text { \# of Coils } & 16[8 \mathrm{radial}] \\ \mathrm{dp} / \mathrm{p} & 15 \% \\ \mathrm{NI} & 5.5 \mathrm{MA}-\mathrm{tums} \\ \text { Stored energy } & \approx 53 \mathrm{MJ} \\ \text { Coil length } & \approx 12.9 \mathrm{~m} \\ \text { Pole area } & \approx 9.7 \mathrm{~m}^{2} \\ \text { Total weight } & \approx 720 \mathrm{tons} \\ \text { Max o.s. radius } & 3.3 \mathrm{~m} \\ \text { Max o.s. length } & 5.0 \mathrm{~m} \\ \text { Overall length } & 9.0 \mathrm{~m}\end{array}$

TABLE II

STAR Operating Characteristics

$\begin{array}{lccccc}\theta_{\mathrm{pq}} & \begin{array}{c}\mathrm{P}_{\max } \\ \operatorname{deg}\end{array} & \begin{array}{c}\Omega_{\mathrm{tot}} \\ (8 \mathrm{gaps}) \\ \mathrm{GeV} / \mathrm{c}\end{array} & \begin{array}{c}\text { Target } \\ \text { Distance } \\ \mathrm{m}\end{array} & \begin{array}{c}\text { Optical } \\ \text { Length } \\ \mathrm{m}\end{array} & \mathrm{D} / \mathrm{M} \\ 5 & 3.07 & 7.4 & 16.0 & 22.8 & \\ 10 & 2.65 & 26 & 7.9 & 15.2 & 5.7 \\ 15 & 2.35 & 46 & 5.2 & 12.8 & 4.8 \\ 20 & 2.15 & 65 & 3.9 & 11.7 & 4.6 \\ 30 & 1.89 & 97 & 2.4 & 10.4 & 4.7 \\ 40 & 1.73 & 125 & 1.7 & 9.7 & 5.2 \\ 50 & 1.64 & 153 & 1.2 & 9.2 & 6.0\end{array}$




\section{Physics Topics}

\section{A. Investigation of the $\mathbf{N} \rightarrow \Delta$ Transition}

The extraction of quadrupole amplitudes in the nucleon-to-delta transition is discussed in the STAR Letter of Intent as a specific example of studies of electroproduction of baryon resonances. Ultimately this project would be expanded in scope to include studies in the first and second resonance regions, for instance, of the $P_{11}(1440)$ Roper resonance, the $S_{11}(1535)$, and the $\mathrm{F}_{15}(1688)$. The reactions $p\left(e, e^{\prime} p\right) \pi^{0}, p\left(e, e^{\prime} \gamma\right) p$ and $p\left(e, e^{\prime} \pi^{+}\right) n$ could be studied with the STAR. The approach advocated by the proponents of STAR would emphasize high precision measurements over a wide range of $\mathrm{Q}^{2}$ of seversl interesting asymmetries. The ability to measure out-of-plane will permit information on the fifth response (TL) to be used in association with the third response (TL), which can be obtained with in-plane experiments, to help in separating the resonant contributions $\left(\mathrm{M}_{1+}\right.$ and one of the desired quadrupole amplitudes $\left.\mathrm{S}_{1+}\right)$. While information should be forthcoming from experiments at medium-energy facilities (Bates, Mainz) in the near term at low $Q^{2}$, and from CEBAF either using polarimetry at modest values of $Q^{2}$ in Hall A or large acceptance coverage with the CLAS detector over a wide range of $Q^{2}$, the strength of the STAR capabilities would be the ability to measure small asymmetries over an extended range of $Q^{2}$.

1. General comments on the relationship of STAR to the rest of the CEBAF program for $N \rightarrow \Delta$ measurements:

The main issue in considering this aspect of what STAR may be able to do centers around the uniqueness of the information which it may provide. The CLAS detector will also address some of the same underlying physics as STAR and, specifically, should also yield important new information on the quadrupole amplitudes for the $N \rightarrow \Delta$ transition over an extended range of $\mathrm{Q}^{2}$. It is not clear to the Subcommittee whether the two detectors are comparable in their ability to extract the quantities being sought (that is, the resonant cor tributions including the quadrupole amplitudes), or whether there is some significant advantage for one device over the other. It is unfortunate that the STAR approach is, in contrast to CLAS, upparently insensitive to the resonant electric quadrupole $E_{1+}$ amplitude. At this point in time, having only the data obtained two decades ago and the various analyses which have attempted to extract the resonant pieces from these old measurements, it is quite hard to evaluate what the near term experiments may provide and what the first generation CEBAF program (experiments using polarimetry in the Hall A spectrometers and using the CLAS) might yield. It is doubly difficult to state with any confidence, what the importance of new data from STAR might be. 


\section{Specific issues:}

An important part of extracting resonant contributions from non-resonant background contributions involves being able to make an isospin decomposition. CLAS has a strategy to do so, however, only two of the three requisite reactions have been discussed for STAR. What is the likelihood of being able to undertake a complete deromposition with STAR?

The analyses presented thus far by the proponents of STAR have involved making the common assumption that only S- and P-wave amplitudes are involved. Moreover they assume that only pieces of the cross section winch involve the dominant $\mathrm{M}_{1+}$ contribution, either alone or when it interferes with some other (small) amplitude, are kept. It is likely that, at the values of $\mathrm{Q}^{2}$ which are accessible at CEBAF, these assumptions are not very good. It is therefore difficuli to extract the desired resonant contributions without having rather complete kinematic coverage of the appropriate observables together with the necessary isospin decompositions. Can STAR provide this coverage and, if so, will it provide the information necessary to execute such an extraction? The more complete set of observables which might be available using polarimetry, for instance, seems to hold promise for getting at the resonant contributions. These appear unlikely to be accessible at high $Q^{2}$ and so the unique power of STAR for such studies appears to be the ability to do high precision measurements of ' ime specific small asymmetries at high momentum transfer. How important is the information which could te obtained this way? Lacking even the near-term measurements at low $Q^{2}$ it is difficult to know what impact such second-generation experiments with STAR could have.

\section{Technical issues:}

As in many of the other STAR experiments, small asymmetries measurements are required and the question of precise relative normalizations must be addressed. We note that the constrained final state in this reaction alleviates some problems such as segment-to-segment acceptance matching that are present in few-body (e,e'p). The error band reportec for $A_{L T}$ included only STAR's pointing errors; it is important to access the total expected error and the way this translates into uncertainty in the extracted $S_{1}+M_{1}$ interference term.

\section{Conclusions:}

While holding promise for high precision measurements of asymmetries involved in the $N$ $\rightarrow \Delta$ transition, including observables which require out-of-plane coincidence experiments and their extension to high $\mathrm{Q}^{2}$, it is hard to evaluate the relative imporance of such studies with STAR. It will be much easier to assess what should be the appropriate thrust of this second-generation program when results are available from medium-energy facilities in the near term and from the first-generation measurements which will be undertaken at CEBAF. 


\section{B. Hyperon Production in the (e,e'K) Reaction}

\section{Physics Area definition}

The (e,e'K) experiments discussed in the STAR Letter of Intent involve several components: threshold measurements requiring out-of-plane capability (to define the real and imaginary parts of the limited number of multipole amplitudes which occur); studies of final-stateinteractions involving strange particles $\left(\mathrm{K}^{+} \mathrm{Y}^{*}, \mathrm{Y}^{*} \mathrm{~N}\right)$; possibly form factors of strange mesons and hyperons; $Y^{*}$ spectroscopy; studies of light hypernuclei. Some of these exploit the capabilities of STAR for example when high luminosity, reasonably large solid angle, out-of-plane measurements are at a premium. For instance, the theshold production measurements require the determination of both real and imaginary pars of specific longitudinal-transverse interferences (he 'TL and TL' responses, respectively). From such measurements it should be possible to extract final-state phase information for the $\mathrm{K}^{+} \mathrm{Y}^{*}$ system and perhaps to address questions relating to PCAC in the strarge sector.

\section{Relation to CEBAF proposais}

CEBAF presently has a number of approved experiments which will address :ome of these physics issues using the HMS/SOS combination or using the CLAS detector. Furthermore, in the longer term the possibilities of undertaking high resolution hypernuclear studies (which neither the STAR nor any approved equipment at CEBAF will be able to approach) will have to be addressed. Consequently it is not clear where the real advantages for STAR lie or whether the existing program will already be able to address the same issues. The part of the Letter of Intent dealing with strangeness physics, while interesting in general terms, lacked sufficient detail to permit a detailed review. Without thorough modeling it is difficult to determine the role of STAR in the context of the approved CEBAF program in strangeness. This is especially true in that STAR would only be implemented as a second generation instrument in a field where essentially no first generation experiments have been done.

The idea of using a limited number of multipoles at threshold together with determinations of the real and imaginary parts of the transverse-longitudinal responses to obtain information on the phase information in the reaction is an intere:sting one. Is it clear that related information could not be obtained using the polarization of the $\Lambda^{0}$ as suggested in some of the approved CEBAF experiments? For most of the rest of the broad program which was outlined, insufficient details were given to be able to identify the relevant physics issues.

\section{Issues}

Studies of this particular reaction may allow one access to a number of interesting and topical physics issues. Nevertheless, no single experiment has actuaily been worked out in 
sufficient detail to be able to evaluate in anything but a rather superficial way, the relative merits of Lising the STAR rather than other devices already in the base plan for CEBAF. For example, the sensitivity of the asymmetries to the difference in the phase shifts could be very useful; however, the size of the signal expected on the basis of existing knowledge was not presented. In order to evaluate this ixperiment, a simulation of the measurement is needed in which the mapping of the phase shifts into measured asymmetries is thoroughly worked out.

The emphasis on the threshold region is driven by the desire to limit the number of partial waves that contribute to the cross section. A quantitative evaluation of the choice of mass region, the corresponding (e,e'K) cross section and an estimate of the amplitudes contained in the first few partial waves is needed. Just how close to threshold does one have to go in order to be able to neglect the higher partial waves? How small is the cross section in this region?

A number of technical design issues need clarification. These are primarily related to the need for forward geometry. Just how is one able to bring HMS or SOS into these small angles? The open geometry of SOS and its limited momentum range make it unattractive. On the other hand, HMS would not be able to reack: the required forward angles. Can the HMS magnet be rotated? How can this be accomplished in view of the fixed rail support system embedded in the floor of the experimental hall? In summary, it is not clear how this measurement is feasible in the geometry required.

Small angles also force the adoption of the invasive geometry in STAR. With STAR aligned along the $q$ vector, it is necessary to transport both the beam and the scattered electron through the in-plane gap. This requires removal of that detector package and insertion of shields in the gap. Can this be done successfully in the space available? How does this impact the two nearby out-of-plane gaps? Hence, how is the symmetry of STAR impaired by this modification?

\section{Conclusions:}

While STAR appears to have a role to play in those aspects of strangeness electroproduction physics where high luminosity, large solid angle, out-of-plane measurements are needed, it does not appear to provide i conpelling motivation for the STAR detector. In the light of the approved program of kaon production at CEBAF, it appears that the STAR detector provides incremental information rather than breaking truly new ground. After the first generation experiments have commenced and yielded the first data in this sub-field, it may be appropriate to proceed in the directions that a device of the STAR type would provide. 


\section{Investigation of Few-Body Systems with the (e,e'p) Reaction}

\section{Physics area and relation to CEBAF proposals}

Studies of the (e,e'p) reaction on the few-body systems could be an important component of the core physics program for both STAR and CEBAF. The proposed measurements in STAR involving separation of several structure functions [including, for example, the out-of-plane ones, $R_{T T}$ and $R_{L T}^{\prime}$, for $500 \leq \mathrm{P}_{r} \leq 1000 \mathrm{MeV} / \mathrm{c}$ and $\left.1 \leq \mathrm{Q}^{2} \leq 4(\mathrm{GeV} / \mathrm{c})^{2}\right]$ could provide strong constraints on the physics content of the (e,e'p) reaction including the reaction mechanism, the high momentum content of the ground state wave function and the final state interactions. A multitude of observables, measured over a large kinematic range, will be necessary to disentangle the various ingredients and STAR could play a role in such studies. The choice of $x>1$ kinematics is an appropriate starting point. Such experiments represent a significant extension to higher $P_{r}$ of the in-plane measurements performed at existing laboratories that were just barely able to probe the regime of recoil momenta $>500 \mathrm{MeV} / \mathrm{c}$. Similar large values of $P_{r}$ will be explored in Hall A, but large solid angle would be necessary to simultaneously study large $P_{r}$ and high $Q^{2}$.

\section{Issues}

At some kinematics, certain of the asymmerries to be measured are rather small and impose demanding requirements (few \%) on relative segment-to-segment normalizations and efficiencies. Methods are needed for dealing with the rapid, and different, cross section variations in different segments along with definition of the precision with which the phase space sampled by different segments must be located (i.e. the definition of which acceptance bins are in common). A realistic estimate of the overall error in the asymmetries (and especially, the physical quantities of interest) would have to be developed and should include resolution and pointing errors of the electron spectrometer. A more developed plan for calibration and monitoring of STAR would be necessary before these issues could be understood.

\section{Conclusions}

The proposed experiments are well-matched to the capabilities of CEBAF and STAR, which by virtue of its large solid angle and high luminosity capability, seems to be an optimal device for extending these studies to extreme values of recoil momentum and $Q^{2}$. CLAS, due to its luminosity limitations, cannot be use'd effectively for measurements of the low rate aspects of the $\left(e, e^{\prime} p\right)$ reaction. There is some overlap with the Hall A progrs $m$ at smaller recoil momenta, but complementary sets of observables would be measured. The Subcommittee concluces that STAR has the potential to make important and unique contributions in this area of physics, and that STAR 
(or some device like STAR) could be an impcrtant additional instrument for second generation experiments at CEBAF.

\section{Nuclear Structure Studies with the (e,e'pp) Reaction}

\section{Physics area and relation to CEBAF proposals}

For many years, the $\left(e, e^{\prime} 2 p\right)$ reaction has been identified as a potentially important new probe of nuclear structure. Important properties of nuclei and nuclear matter such as two-body densities and three-body forces can, in principle, be addressed by this technique. It is equally clear that this will not be easy. Unambiguous extraction of the physics will involve a massive effort by both experimentalists and theorists in the years to come. One can draw a parallel with the development of the (e,e'p) reaction over the last two decades.

\section{Issues}

At STAR, it will be possible to explore one specific aspect of the reaction where the two protons are emitted in "perpendicular kinematics". The advantages of this kinematical arrangemint over two other choices are clearly laid out in the proposal. It is not obvious, however, that this is the most important obstacle in extracting the physics. For example, final state interactions can change the cross sections by orders of magnitude as illustrated in the calculations presented.

It appears that the experiment is technically feasible at least for the ${ }^{3} \mathrm{He}$ case. However, the 3 He case is rather special and it is unclear how feasible this approach is for heavier systems.

\section{Conclusions}

This is an interesting application of the STAR that addresses an important new application of electron scattering and which will be complementary to the low luminosity, survey work that will be possible at CLAS. However, we see this as an experiment rather than a program. A full program to address this important physics topic would need to probe a much larger part of the phase space for the reaction and be capable of studying a variety of nuclei.

\section{E. Measurement of GEn in a Recoil Polarimetry Measurement}

\section{Physics area}

No oral presentation was made for an experiment using STAR to measure recoil nucleon polarization, but the possibility of measuring $G_{E n}$ in a polarization tansfer experiment was described in the written documents. In this method the polarization of recoil neutions from the scattering of polarized electrons at the quasi elastic peak in deuterium would be measured by scattering the recoil neutrons from a liquid hydrogen analyzer. The STAR spectrometers would be used to measure the azimuthal distribution of protons recoiling from n-p scattering in the analyzer. 
The advantage of STAR for this experiment is the combination of large acceptance for proton detection over a range of azimuthal angles, together with good momentum resolution so as to isolate the elastic $n-p$ scattering from backgrounds and to maximize the analyzing power. The raw asymmetry is expected to be small [around $5 \times 10^{-3}$ at $\mathrm{Q}^{2}=0.15 \mathrm{GeV} / \mathrm{c}^{2}$ ]. The statistical precision of $0.15 \%$ required for a $15 \%$ to $20 \%$ measurement of $G_{E n}$ at $Q^{2}=0.12$ to $0.29 \mathrm{GeV} / \mathrm{c}^{2}$ is estimated to be obtainable in 3 to 4 days. Systematic errors in the azimuthal asymmetries would also have to be kept to the level of $0.15 \%$ or better. The proponents did not indicate how this would be done. However, in contrast to the other applications of STAR for measurements of recoil proton azimuthal asymmetries around the q-vector, there are several advantages in this case. For example, the particle rates in the various STAR segments will be similar, making relative detector efficiencies easier to understand and control. It is also possible to make measurements with the beam polarization set to zero or reversed for calibrations. There may be some problems making measurements at $Q^{2}$ below $0.2 \mathrm{GeV} / \mathrm{c}$ where there will be large effects from multiple scattering and energy loss of the low momentum protons $(0.1$ to $0.2 \mathrm{GeV} / \mathrm{c})$ in the analyzer and detector maierials.

\section{Relation to CEBAF Proposals}

In competition with this proposal, there is an other experiment (EXP 89-05) approved conditionally by PAC4 for Hall A to measure GEn using a solid scintillator system as a recoil neutron polarimeter and another deferred experiment (EXP 89-18) to measure $G_{E n}$ in a similar momentum range $\left(0.5 \leq \mathrm{Q}^{2} \leq 2.0[\mathrm{GeV} / \mathrm{c}]^{2}\right)$ using polarized electrons scattering from polarized solid targets in Hall $\mathrm{C}$. Comparisons of the counting rates indicate that the Hall $\mathrm{A}$ experiment and the STAR proposal have similar counting rates at $Q^{2}$ around $0.2(\mathrm{GeV} / \mathrm{c})^{2}$. There will be other differences in sensitivity due to the different effective analyzing powers of the two methods, and different systematic errors due to various instrumental effects. While none of these factors have been worked out in detail, they are probably comparable for the two experiments.

\section{Conclusions}

Given that the Hall A experiment will probably be performed before the STAR experiment, and that the STAR proposal does not have a significant ad antage in counting rate sensitivity over the Hall A experiment, the STAR spectrometer does not offer a unique advantage either in kinematic range or in precision for measurement of $\mathrm{GEn}_{\mathrm{n}}$.

\section{F. Parity Violation Measurements}

\section{Physics area}

Parity violation experiments at $\mathrm{CEBAF}$ have the potential to explore some of the most fundamental physics which can be addressed with several $\mathrm{GeV}$ high intensity beams of polarized 
electrons. The possible focus includes high-precision tests of the electroweak interaction itself and studies of hadronic form factors of the nucleon, together with measurements of weak interaction nuciear response functions. Important amongst these is the determination of the strangeness and isospin content of the nucleon.

Several specific experiments have been discussed in general terms, including: elastic scattering from the proton (strangeness radius determination); deep-inelastic scattering to determine the quark distributions in hadronic systems; elastic and inelastic scattering from nuclei to probe the isospin, strangeness and nuclear structure (this entails using specific good quantum numbers of nuclei to "filter" specific pieces of the electroweak currents); and baryon excitations such as the axial-vector contribution in the $\mathrm{N}$-to-delta transition.

CEBAF may be superbly suited to such a program as it will have high quality, high intensity beams of polarized electrons. From experience obtained at SLAC, Mainz and Bates in previous parity violating electron scattering experiments it should be possible to have excellent control over systematic errors -- something which is critical in measurements of the very small asymmetries such studies entail. For a major program of parity violation measurements it will also be necessary to have a large acceptance magnetic spectrometer capable of handling the extreme luminosities required to accumulate significant statistics in a reasonable amount of time. Also attractive, but perhaps not critical for such parity experiments, is azimuthal symmetry. These attributes are provided by the STAR detector, although other specially tailored devices may equal or exceed the capabilities of STAR as a multi-purpose instrument.

In general terms the Subcommittee is enthusiastic about the potential value of making a commitment to undertake parity violation experiments at CEBAF. The study of elastic scattering from hydrogen in particular was seen as one strong possibility. A detector of the STAR type could provide an important capability in this regard, namely for the study of high precision parity violating electron scattering at small angles.

\section{Relationship to the rest of the CEBAF program}

Although there are no approved experiments on parity violating electron scattering at CEBAF, the PAC has followed with interest the efforts of the CEBAF scientific staff and the parity community in general to develop a detailed plan for undertaking such a program. Specifically, in the report of PAC4, the Committee identified the importance of making sure that the beam quality provided by the accelerator be sufficiently well controlled that such very small asymmetry measurements could be accomplished when the time is appropriate. 


\section{General issues}

The Subcommittee was disappointed in the level of detail presented conceming parity experiments at CEBAF, either using STAR or using some other instrument. The design work on STAR itself was at a much greater level of sophistication than any modeling of a potential experiment which might use this device. If one of the central roles for STAR was to serve the needs of parity violation experiments at CEBAF, then a much more convincing case would have to be made. The presentation itself focused on parity violation in delta production, an idea which was not well developed either in theory or experiment. For example, the diagram of the apparatus which was presented seemed to be inconsistent with what was shown for the basic design of STAR. It was difficult to assess what problems exist for elastic proton scattering.

\section{Specific issues}

A number of key issues were not addressed:

a. Should the experiment be performed at different angles in order to isolate the three accessible form factors, or should some input from theory be used to extract the most crucial information?

b. Are there other instruments that could equally well make the measurement? For example, how significant a measurement can be made with conventional spectrometers? To justify a project with the cost projected for STAR, a solid study of the best that one can do with alternative approaches is appropriate. While this may not be the responsibility of the STAR designers, many of the principals in parity violating electron scattering studies are listed on the Letter of Intent, and the community turns to them for an analysis of this issue.

\section{Conclusions}

Although the Subcommittee viewed with great interest the prospects for parity violation experiments at CEBAF, it has nevertheless reached the conclusion that the case for using STAR was not developed in sufficient detail to help justify the cost of this large project. 


\section{STAR Design and Performance}

\section{A. Concept and Specifications}

These considerations have been addressed thoroughly in the presentations and documentati in and are summarized briefly in Section II.

\section{B. Issues}

\section{Magnet}

The toroidal array of eight spectrometers appears to be a viable geometry which could be constructed and meet the desired specifications. However, there are many details that would need to be worked out during the conceptual design and initial engineering stages. Some issues which would need to be addressed are:

a. Is the initial choice of $20 \mathrm{kA}$ operating current really optimum in terms of overall cost including power supply and heat load, and is it balanced relative to the turn number? (Issues such as making the 24 coil-coil cross-connects with $20 \mathrm{kA}$ conductor and the possible field perturbations need to be addressed. Also 3 pairs of $20 \mathrm{kA}$ leads require about $500 \mathrm{~W}$ of refrigeration capacity to cool them; this is the penalty for returning the gas at room temperanure rather than cold.)

b. Quench calculations for a cryostable coil are tricky. A discussicin of the assumptions made and whether they are realistic is needed.

c. When the supertube is used in the invasive geometry, it will influence the field distribution in the adjacent gaps. The size of this effect should be estimated.

d. Complete 3D calculations may be used to address item c) as well as to confirm the overall magnet field integral specifications.

e. It may be that the field flatness specifications of one part in a thousand is tighter than necessary because the particle envelope for any given initial drift distance only occupies a small fraction of the dipole width. Obviously, some radial gradient is tolerable and allowing it may simplify the multiple coil structure.

2. Optics

The design of the spectrograph optics of the individual gaps and the variation of the matrix elements with drift length seems reasonable. In fact, the resolution specifications for the short drift length appear to be too good. With three times the solid angle per gap and $50 \%$ more momentum range than HMS, the resolution is nearly the same. This claim should be justified with more detailed higher-order calculations. 


\section{Calibration}

Assuming that the questions about mechanical stability and sproducibility raised below can be answered satisfactorily, another major calibration issue would still remain to be addressed. During asymmetry measurements, there will be large differences in the singles rates and the associated real/accidental ratios, due to the primary scattering angle differences of the gaps. This will make the extraction of the coincidence cross section differences at the $1 \%$ level very difficult.

\section{Mechanical}

There are several important issues related to mechanical systems:

a. Is the mechanical rigidity of the frame or alignment system adequate for maintaining the pointing accuracy as the initial drift distance is varied, i.e. when the 800 ton system is moved back and forth? To answer this it would be necessary to consider floor deflections, beam deflections, active alignmen: system, etc. If an active alignment or point system such as for HRS is envisioned, then the cost of the carriage will be greater than that for the HRS, since the mass of STAR is greater, while the pointing requirements are essentially the same.

b. The details of the scattering chamber/pivot point area may be complicated when various requirements of the HMS, SOS and STAR are considered. A discussion of what is involved and how time consuming it is to move the STAR to 0 or 180 degrees is needed.

c. For special experiments which require both HMS and STAR to be at very small angles, the interference may make it necessary to pivot HMS about its rear end and move the scattering target upstream. This seems possible but only if some new mechanical features are built into the HMS support structure before it is too late.

\section{Extemal fields}

The impact of the relatively large external fields on some aspects of the proposed experiments would have to be addressed in detail. For example, how will these fields influence beam and scattered electron trajectories? polarized beams?, targets?, etc? Will a nearby large iron mass such as an HMS perturb the STAR gap fields and thereby introduce systematic errors which depend on relative spectrometer positions? 
In summary, the initial concept of the STAR appears feasible, but there are many important issues which would have to be clarified before detailed magnet and mechanical designs could be considered final. Some optical issues would need to be further documented, the effects on calibrations of unequal singles rates in the gaps would need to be studied, and several issues related to mechanics and effects of external fields would need clarification.

\section{Costs, Manpower, and Schedule}

Although the cost estimate presented was not reviewed in detail, the number presented, $\$ 16 \mathrm{M}-\$ 18 \mathrm{M}$, appears to be reasonable for this stage of the project. Adding $30 \%$ contingency, the Subcommittee concludes that $\$ 20-22 \mathrm{M}$ is a good working estimate for the final cost of the project. However, this estimate has been made before a serious engineering design has been completed. Experience shows that costs for projects such as this inevitably rise rather than fall with time.

The cost versus performance trade-off table may be reasonable for small excursions about the design presented, but the Subcommittee was not convinced that this table represented what would be achievable in physics performance if a detector had to be constructed for considerably less money.

The schedule presented seems reasonable for a measurement program beginning in 1998 if funding were to start in early FY 1995.

The manpower committed to the project is obviously too small. The collaboration is well aware of this and we do not see this as necessarily a major concern at this time. We believe that ultimately the manpower needed would be comparable to that in Hall A (approximately 150 FTE).

\section{Summary and Conclusions}

\section{A. Specific Physics Areas}

\section{Investigation of the $N \rightarrow \Delta$ Transition}

STAR offers promising and to some extent unique opportunities to perform high precision measurements of asymmetries involved in the $\mathrm{N} \rightarrow \Delta$ transition, including observables which require out-of-plane coincidence experiments and their extension to high $Q^{2}$. However the Subcommittee found it hard to evaluate the impact of such studies with STAR so far in the future and before the next series of measurements are complete. It will be much easier to assess what should be the appropriate thrust of this second generation program when results are available in the 
near term from experiments at medium-energy facilities and then from the first generation measurements which will be undertaken at CEBAF.

\section{Hyperon production in the (e,e'K) reaction}

While the STAR facility appears to have a role to play in those aspects of strangeness electro-production physics where high luminosity, large solid angle, out-of-plane measurements are needed, it does not appear to provide a compelling motivation for the STAR detector. In the light of the approved program of kaon production at CEBAF, it appears that the STAR detector would provide incremental information rather than breaking truly new ground. After the first generation experiments at CEBAF have commenced and have generated new data in this subfield, it may be appropriate to proceed in the directions that a device of the STAR type would emphasize.

\section{Investigation of few body systems with the (e,e'p) reaction}

The proposed measurements on few body systems are well matched to the capabilities of STAR. By virtue of its large solid angle and high luminosity capability, STAR seems to be an optimal device for extending these studies to extreme values of recoil momentum and $Q^{2}$. There is some overlap with the Hall A program at smaller recoil momenta but complementary sats of observables would be measured. Low rate measurements of (e,e'p) at CLAS are difficult due to its luminosity limitations. The Subcommittee concludes that STAR has the potential to make important and unique contributions in this area of physics and that STAR (or some device like STAR) could be an important additional instrument for second generation experiments at CEBAF.

4. Nuclear structure studies with the (e,e'pp) reaction.

An investigation of very light nuclei with the (e,e'pp) reaction in perpendicular kinematics is an interesting application of STAR. It addresses one aspect of a new subfield of electron scattering which will develop at the new CW accelerators and which can potentially provide information on important topics such as two-body densities and three-body forces in nuclei. Because of the rather limited nature of those specific measurements which can be carried out at the proposed facility, the Subcommittee views this as an important and interesting experiment at STAR rather than as one of the major programs for this device.

\section{Determination of $G_{E n}$ in a Recoil Polarimetry Measurement}

This measurement is in direct competition with experiment 89-005 in Hall $\mathrm{A}$ in which a solid scintillator system is used for a recoil neutron polarimeter. In the Subcommittee's judgement the STAR spectrometer does not offer a unique advantage either in kinematic range or in precision for the measurement of $\mathrm{GEn}_{\mathrm{n}}$ compared to other methods. 


\section{Parity Violation Measurements}

The Subcommittee viewed with great interest the possibility of exploring weak interaction physics at CEBAF though a number of parity violation measurements. In the Letter of Intent emphasis was placed on the elastic scattering of polarized electrons from hydrogen and on the $\mathrm{N} \rightarrow$ $\Delta$ transition. It is the Subcommittee's judgement that the case for using STAR for these parity violation measurements was not developed in sufficient detail in order to help justify the cost of this large project.

\section{B. STAR Design and Performance}

It is the judgemert of this Subcommittee that the initial concept of STAR is feasible and could serve as the basis for a more detailed design. However a number of very important issues related to the design and calibration of STAR need to be clarified and documented.

\section{Costs, Manpower, and Schedules}

The cost estimates provided for STAR plus $30 \%$ contingency bring this project into the $\$ 20 \mathrm{M}$ to $\$ 22 \mathrm{M}$ range. Without modification of the design goals it is not likely to be less.

The Subcommittee estimates that the manpower requirements of this project would be comparable to those of Hall A, i.e about 150 FTE.

The schedule presented requires funding to start in early FY 1995 in order to begin a measurement program in FY1998, as proposed.

\section{Summary}

The major thrusts of the ST'AR program are the investigation of spin dependent and out-of-plane structure functions and the investigation of parity violation phenomena.

At CEBAF, the out-of-plane coincidence program of STAR complements the programs planned for Hall A using polarimetry or the proposed MPS facility, and for Hall B using CLAS. STAR, with its large acceptance and high luminosity capability, is unique in its ability to study small effects at high $\mathrm{Q}^{2}$ and at large recoil momentum. However, the new generation o experiments now planned or in progress at Bates, at Mainz, and developing at CEBAF have the potential for generating major advances in our understanding of spin dependent and out-of-plane structure functions. Thus it is difficult to assess, at this time, the incremental impact of the additional information provided by STAR. It is the Subcommittee's judgement that major additions to the CEBAF out-ofplane capability should await developments in the programs at the lower energy facilities. 
The investigation of parity nonconserving processes is potentially a very interesting new component of the CEBAF physics program. Although several experiments have been discussed, a specific physics goal for the development of this program has not been established since no clear path to a precision measurement has yet evolved. The relative merits of a program using STAR, the Hall A spectrometers, or a new magnetic device (for example a solenoid) have been discussed but not worked out. It is the judgement of this Subcommittee that the case for basing this weak interaction physics program on the STAR facility has not been made.

\section{E. Subcommittee Recommendation}

It is the unanimous conclusion of this Subcommittee that, as presented, the incremental physics impact of the STAR program over experiments in progress or planned, is not sufficient to justify an expenditure which we judge to be in excess of $\$ 20 \mathrm{M}$. We do not recommend construction of this device.

The Subcommittee gave some thought as to the merits of focusing on a more limited physics program based on a less expensive but more limited spectrometer. There was no consensus among the Subcommittee members as to what physics could serve as the basis for such a more limited design and we take no position on this issue. 


\title{
APPENDIX A
}

\section{MEMBERSHIP OF THE PACA SUBCOMMITTEE FOR THE REVIEW OF STAR}

Members

\author{
Dr. Raymond G. Arnold \\ American University \& SLAC \\ Dept of Physics \\ McKinley 102 \\ Washington, DC 20016 \\ Dr. Peter D. Barnes ( Chainnan ) \\ Physics Department \\ Camegie Mellon Univ. \\ Forbes Ave. \\ Pittsburgh, $\mathrm{Pa} .15213$ \\ Dr. T. William Donnelly \\ Center for Theoretical Physics \\ M. I. T. 6-300 \\ Cambridge, Mass., 02139 \\ Dr. T. James M.Symons \\ Nuclear Science Div. \\ Lawrence Berkeley Lab \\ 1 Cyclotron Rd. \\ Berkeley, Calif., 94720
}

Consuitants

[r. Robert Lourie

Physics Dept.

Univ. of Virginia

Chariottesville, Va. 22901

Dr. Jerry A. Nolen

Dept. of Physics and Astronomy Michigan State Univ.

East Lansing, Mich. 48824

Dr. Paul Souder

Dept. of Physics

Syracuse Univ.

Syracuse, N.Y. 13244 


\section{APPENDIX B CHARGE TO THE SUBCOMMITTEE}

The following charge from the CEBAF management to the PAC was conveyed to the Subcommittee.

The STAR is being designed to support a program of research that includes out-of-plane coincident $\left(e, e^{\prime} p\right)$, parity violation and (e,e'2p) experiments. It may also be useful for recoil polarimetry, $\left(e, e^{\prime} K\right)$, and backward angle $\left(e, e^{\prime}\right)$ measurements. Assume that an instrument with roughly the STAR's design specifications can, in fact, be built. Is the physics program sufficiently interesting to justify its construction? In particular, is the STAR the right approach to doing this kind of physics at CEBAF, and is it sufficiently complementary to other instruments in the CDR? For the sake of this discussion assume that the construction cost of the STAR will be in the neighborhood of $\$ 16 \mathrm{M}$, and it will take an effort comparable to HMS plus the SOS to construct it.

What major uncertainties do you perceive in the STAR's capabilities to carry out the planned physics program? Here the PAC ( Subcommittee) should focus on inherent high-level problems such as the pointing errors and calibration identified at PAC III, rather than on technical details in the design of the instrument. 
APPENDIX C

APPENDIX C

AGENDA OF THE MEETINGS AT CEBAF

JULY $17-18,1990$

Room L104/L102 CEBAF Center

TUESDAY, JUY 17

8:00 Executive Session

8:40 OPEN SESSION

J. D.Walecka, Welcome

J. Domingo, STAR Subcommittee Charge

9:00

L. S. Cardman STAR - Overview

V.R. Pandharipande Theoretical Perspective

10:15 Coffee

10:30 C. N. Papanicolas N- $\Delta$ Transitions
A. M. Bernstein
$\mathrm{H}\left(\mathrm{e}, \mathrm{e}^{\prime} \mathrm{K}\right)$
A. Magnon
Few Body Physics
$25 \mathrm{~min}$
$25 \mathrm{~min}$

$30 \mathrm{~min}$

$20 \mathrm{~min}$

$25 \mathrm{~min}$

12:30 Lunch / Executive Session

2:00

R. Alarcon

(e,e'2p) / Correlations

$25 \mathrm{~min}$

B. Mecking

Remarks

$20 \mathrm{~min}$

R. Lourie

Remarks

$20 \mathrm{~min}$

3:40 Coffee

4:00 R. Laszewski

4:45 Executive Session

Working Dinner

Wednesday, July 18

8:30 R. Laszewski

J. Napolitano

P. Souder

C. N. Papanicolas

10:30 Coffee

10:45 Executive Session

Working Lunch

4:30 Close Out

5:00 Adjournment
STAR Design and Performance $\quad 30 \mathrm{~min}$

Schedule \& Costs $20 \mathrm{~min}$

Parity Violation Experiments $25 \mathrm{~min}$

Remarks $20 \mathrm{~min}$

Concluding Remarks $20 \mathrm{~min}$ 

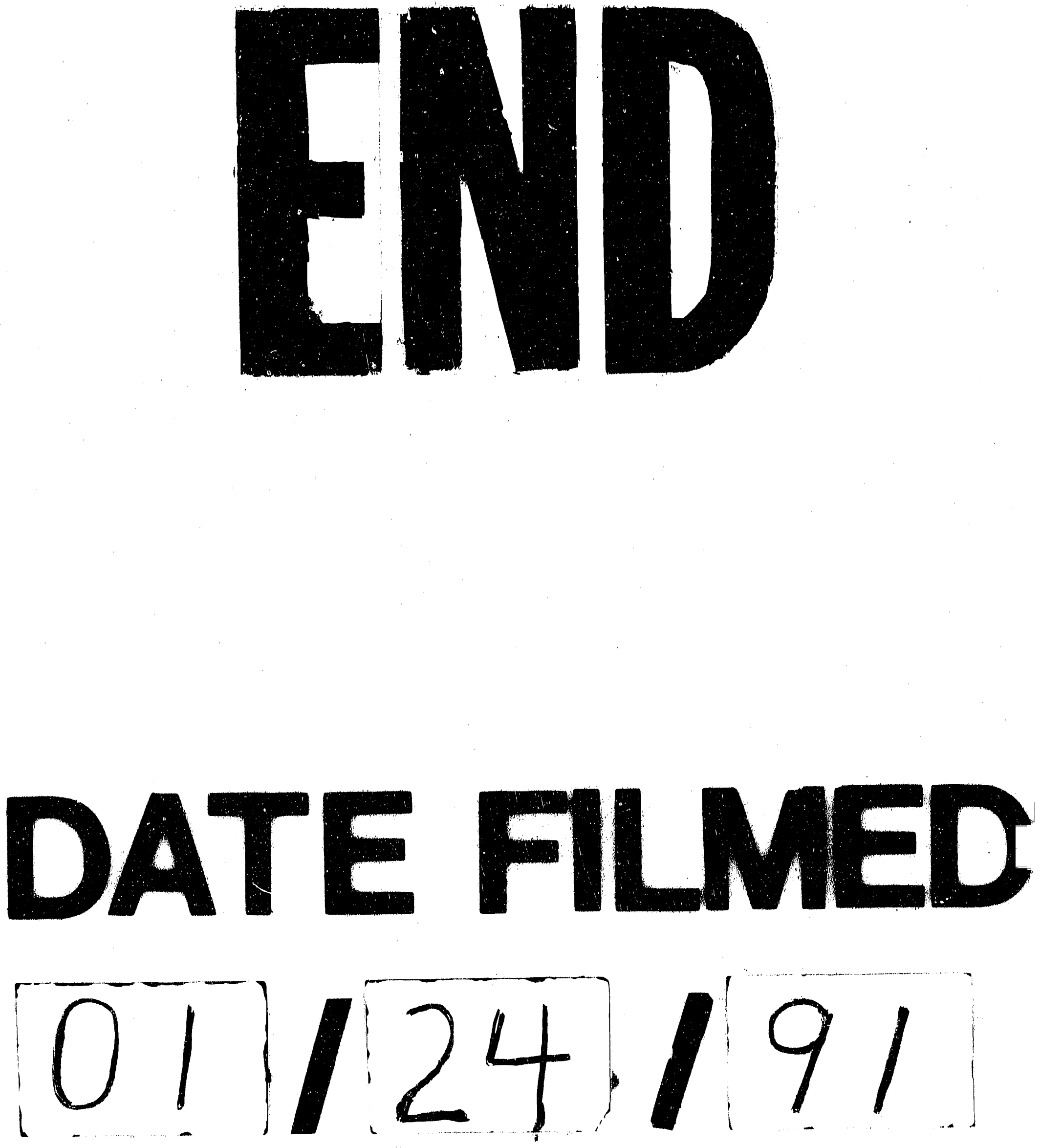
